\title{
Microsurgical Management of Neovascularisation Secondary to Posterior Segment Ischaemia
}

\author{
DAVID McLEOD
}

Manchester

\begin{abstract}
Summary
Structural, metabolic and functional rehabilitation of eyes affected by the haemorrhagic and tractional sequelae of neovascularisation secondary to posterior segment ischaemia is discussed. Microsurgical management must pay due attention not only to the mechanical but also to the underlying cell-biological implications of the surgical pathology. The appropriateness of case selection for surgery rests upon the likelihood of successful technical and functional outcomes and also upon the overall visual status of the patient, issues of particular relevance to diabetes.
\end{abstract}

The principles underlying the surgical management of the vasoproliferative retinopathies have been substantially expanded and refined in the 15 years since the Cambridge Ophthalmological Symposium on the Vitreous in 1975 . The precise microsurgical implications vary according to aetiology, especially in respect of the site of neovascularisation (e.g. peripherally in sickle cell retinopathy and retinopathy of prematurity, mid-peripherally in Eales' disease or mid-peripherally and centrally in diabetes mellitus). However, vitrectomy for the haemorrhagic and tractional sequelae of proliferative diabetic retinopathy is archetypical and remains one of the most frequent and indeed the most effective types of vitreous surgery.

With modern financial restraints on the overall provision of health care (perhaps best exemplified in countries like the UK which seek to provide comprehensive care for the whole population), and with the pressure to focus scarce ophthalmic resources on costeffective procedures such as cataract extraction, it is more than ever necessary to docu- ment and justify diabetic microsurgery not only to one's ophthalmic peers through medical audit but also to governmental bodies and to local managerial and public health professionals to whom responsibility for the detailed distribution of resources is being increasingly devolved. Cost-effectiveness presumes optimal technical and functional outcomes and demands careful definition of surgical objectives together with consideration of the appropriateness of case selection.

\section{Microsurgical Rationale}

The multitude of microsurgical manoeuvres potentially employable in diabetic vitrectomy have, as their underlying themes, both physical and cell-biological purposes, and these objectives cannot be clearly distinguished. Suction-cutting and/or aspiration of haemorrhage readily restores the transparency of the media intra-operatively, but close attention should also be paid to removal of basal haemorrhagic residues (e.g. using scleral indentation) especially recent and only partially lysed clot in the inferior retrolental area. 
Even if the vitreous base is closely cropped with subtotal debulking of haemorrhage, leaching of residual red cells or minor rebleeding can result in prolonged postoperative vitreous haze if the anterior hyaloid barrier to red cell outflow through the trabecular meshwork remains intact. Specific disruption of a localised part of the anterior hyaloid membrane, visualisation of which is often facilitated by crescentic aggregation of erythrocytes in the region of Weiger's ligament, creates a state of 'pseudo-aphakia' and enables any residual red cells to egress readily via the trabeculum. Similarly, careful retrolental vitrectomy precludes anterior haemorrhagic reaccumulation. Eyes successfully rendered 'pseudo-aphakic' demonstrate a similar suspension of red blood cells in the aqueous fluid of the anterior chamber and the retrolental space postoperatively.

Complete removal of the post-basal posterior vitreous cortex, together with any contained fibrovascular tissue, is an aesthetic (if not always quintessential) surgical objective. The relief of anteroposterior, bridging and especially tangential epiretinal traction permits retinal re-attachment and mobilisation of any associated breaks with minimal residual distortion (accepting a degree of surface wrinkling retinopathy). Failure to at least circumcise all fibrovascular epiretinal proliferations is frequently associated with postoperative rebleeding or detachment from retinal break formation. It may also be necessary to remove non-vascularised epimacular proliferations growing independently of an overlying detached vitreous. Retinal breaks require to be sealed by surrounding coagulation and closed by internal tamponade with or (usually) without scleral buckling. Any pars plana incision threatens retinal disruption near the entry sites either intra-operatively or (very rarely) post-operatively, especially via multiple instrument-penetrations of basal vitreous attached to peripheral ischaemic retina. ${ }^{1}$

Removal of vitreous gel improves the movement of oxygen within the vitreous cavity, ${ }^{2}$ and this might be of marginal benefit to the innermost part of the retina and in respect of the 'neovascular activity' of any residual proliferations. ${ }^{3}$ However, the tendency of any residual new vessels to regress and the infrequency of recurrent post-basal vasoproliferation appear to reflect two other properties of the vitreous gel relevant to the vasoproliferative retinopathies. Following inner retinal ischaemia the vitreous gel appears to be 'conditioned' by a variety of macromolecules (including fibroblast growth factor-FGFand endothelial cell stimulating angiogenesis factor-ESAF) analogous to in vitro production of crude retinal extract. ${ }^{4,5}$ The glycoprotein content of the gel is thought to have a role in localising these molecules near the retinal surface, thus facilitating epiretinal vasoproliferation. This 'macromolecular confinement' by gel is illustrated during fluorescein angiography of proliferative retinopathy when fluorescence remains localised for a considerable period to the immediate vicinity of the new vessels. In addition, the collagen component of the gel appears to act as a scaffold or substrate for the microvascular cell proliferation, in the absence of which (e.g. following posterior vitreous detachment) vasoproliferation is restricted to abortive outgrowths ${ }^{6}$ to the optic disc or to the vitreous base region $;^{7}$ in these circumstances, the intraocular movement of molecules is subject to the effects of convectional and saccadeinduced currents. ${ }^{6}$ While complete removal of the post-basal vitreous cortex denies postbasal vascular reproliferation (except in the context of silicone oil injection ${ }^{8}$ ), continuing leaching of growth factors from the ischaemic posterior retina is thought to be responsible for basal and retrolenticular neovascularisation $^{9}$ or rubeosis iridis especially in aphakic (and indeed pseudo-aphakic) eyes via enhanced anterior flow and diffusion of these molecules. However, in all but the most ischaemic diabetic eyes, these postoperative complications can be avoided by scatter photocoagulation provided this is timely (intraoperative endophotocoagulation being the most convenient) and of sufficient extent (coagulation of the mid-peripheral and equatorial retina helping to mitigate subsequent nyctalopia and field constriction). Such photocoagulation also generally prevents retro-silicone oil neovascularisation. The mechanism of photocoagulation's effects remains obscure, whether by increasing inner 
retinal oxygenation, ${ }^{10}$ reducing growth factor production or changing the growth factor 'mix' or 'context'.

Non-vascularised reproliferation also occurs after diabetic vitrectomy especially following silicone oil injection or in the context of a rhegmatogenous postoperative detachment. ${ }^{11}$ In the latter circumstances, the intraocular cell-biology corresponds to that in proliferative vitreoretinopathy, i.e. subretinal fluid is 'conditioned' by secretions from the pigment epithelium and freely exchanges with preretinal fluids via a retinal break. Thus, post-vitrectomy retinal detachment should be avoided at all costs or urgently re-operated. Furthermore, retinal detachment in an aphakic vitrectomised diabetic eye inevitably results in rubeosis iridis and ultimate phthisis.

\section{Materials and Methods}

Based on the above rationale, a consecutive series of 200 primary vitrectomies for complications of proliferative diabetic retinopathy were undertaken between January 1985 and March 1988. Twenty-eight of the 172 patients underwent bilateral surgery, 136 patients were insulin-dependent and there were 99 males and 73 females. Surgery was undertaken by the consultant in charge (DMcL) in 140 procedures, by a vitreoretinal fellow in 50 eyes and by a resident surgical officer in 10 eyes. Sixteen eyes were aphakic, three were pseudophakic and 181 phakic.

In 119 eyes, surgery was undertaken for vitreous haemorrhage either alone (64 eyes) or in combination with extramacular traction retinal detachment (55 eyes). Of the eyes in this haemorrhage group, 24 had rubeosis iridis preoperatively (identified biomicroscopically). Selection of patients with vitreous haemorrhage for vitrectomy did not depend primarily on the duration of haemorrhage but took into account a variety of factors including the presence of rubeosis, traction detachment by ultrasound, prehaemorrhage visual acuity, and the state of the other eye.

In 81 eyes, surgery was performed primarily for macular traction retinal detachment or retinoschisis either alone (22 eyes), in association with vitreous haemorrhage ( 22 eyes) or as part of a combined traction and rhegmato- genous detachment ( 37 eyes). In this macular detachment group of eyes, preoperative rubeosis iridis was present in 12 eyes. Selection of patients with macular detachment for vitrectomy depended on the duration and height of the macular detachment (where this could be defined) together with the predetachment acuity, but only to a limited extent upon the anticipated technical problems in membrane dissection. Eyes with shallow macular detachment, clear media and vision of less than finger counting were generally considered unsuitable for surgery in view of the very poor visual prognosis (the 'finger counting test'), and eyes with a highly elevated macular detachment of more than six months' duration were also generally excluded.

Three-port vitrectomy, intraocular diathermy and membrane dissection by segmentation or delamination was performed as developed and popularised by Charles. ${ }^{12}$ In eyes with extensive vasoproliferation, an 'en bloc' technique was employed as originally described in conjunction with viscodelamination of non-detached vitreous cortex ${ }^{11,13}$ and as subsequently fostered by Abrams. ${ }^{14}$ In haemorrhage cases, phakic eyes were rendered 'pseudo-aphakic' as were pseudophakic eyes which underwent peripheral iridectomy (and focal anterior hyaloidectomy) from behind. In the combined series, viscous separation using $1 \%$ Healon or $1 \%$ Methylcellulose was employed in 24 eyes, internal tamponade using SF6 gas in 92 eyes, prolonged internal tamponade using silicone oil in 38 eyes, simultaneous lens removal in five eyes and peripheral scleral buckling in 30 eyes.

Intra-operative laser photocoagulation was performed to all previously untreated or under-treated retina outside the major vascular arcades and nasal to the optic disc, paying particular attention to the equatorial and post-oral retina. The intensity and density of laser burns was 'titrated' according to the apparent severity of ischaemia, the 'activity' of the posterior neovascularisation, and the presence or absence of preoperative rubeosis iridis. An eye with no previous scatter treatment would typically receive 2,000 endolaser burns. Focal or grid photocoagulation was also directed towards any maculopathy.

The indications for use of silicone oil tam- 
ponade differed somewhat from previous reports ${ }^{15,16}$ reflecting the progress in microsurgical technology since those original descriptions (including better facilities for phakic fluid-gas exchange, endolaser photocoagulation, etc). Silicone oil was injected especially in eyes with multiple pre-existing/iatrogenic retinal breaks, in eyes with very oedematous retina which (even after re-attachment) precluded satisfactory endolaser treatment particularly in the vicinity of breaks, after retinectomy, in anticipation of likely problems with postoperative posturing, or often with a combination of these factors.

Particular attention was paid to examination of the postoral retina in the vicinity of the instrumentation entry-sites at the completion of the microsurgery. Cryopexy was generally performed in these areas irrespective of the discovery of any breaks, and any identified retinal disruption necessitated internal tamponade and also scleral buckling in those eyes with excessive basal gel incarceration.

\section{Results}

Intra-operative complications included iatrogenic breaks in 44 eyes $(22 \%)$, either entrysite related breaks (19 eyes) and/or posteriorly-located breaks (30 eyes); the latter were generally 'accidental'. Breaks at the posterior border of the vitreous base unassociated with retinal detachment and considered to be preexisting rather than iatrogenic were discovered and treated in two eyes. Lens touch occurred in two eyes. No patient died during the postoperative study period.

\section{(i) Technical Outcome}

The surgical results are presented in accordance with a schema employed previously. ${ }^{17}$ The principal objective of microsurgery was stabilisation', i.e. no vitreous haemorrhage, no retinal detachment, no rubeosis (or other vasoproliferation such as retrolental neovascularisation) and no retained silicone oil. Eyes in which stabilisation was achieved at six months postvitrectomy without any further surgical intervention were considered to have been 'immediately stabilised' and therefore to have undergone 'definitive' surgery. The term ultimate stabilisation' referred to eyes which underwent post-vitrectomy intervention(s) such as laser, vitreous washout, silicone oil removal, scleral buckling, etc, but which were nevertheless stabilised six months following such further intervention. Eyes were considered 'unstable' if there was persistent rubeosis iridis or persistent silicone oil in-situ at six months post-vitrectomy. The term 'failed surgery' referred to eyes with retinal detachment, macular distortion, vitreous haemorrhage or rubeotic glaucoma at six months post-vitrectomy.

The results of vitrectomy for vitreous haemorrhage are presented in Figures 1 and 2 which indicate that 98 out of the 119 eyes were immediately stabilised $(82 \%)$. The post-vitrectomy procedures required to achieve ultimate stabilisation in a further 10 eyes included vitreous washout with completion of vitrectomy and endolaser photocoagulation in six eyes (including one eye requiring lensectomy in addition), biomicroscopic laser photocoagulation in two eyes (plus removal of silicone oil in one of these eyes), scleral buckling in one eye and insertion of a Molteno drainage tube in one eye. The three unstable eyes included two with silicone oil present at six months post-vitrectomy and one eye in which gross pre-operative rubeosis could not be completely reversed by intra-operative and post-operative photocoagulation but in which the intraocular pressure was controlled medically. The reasons for failure in eight eyes included retinal detachment in three eyes (all of which developed severe rubeosis), severe rubeosis without retinal detachment in one eye, macular reparative epiretinal fibrosis in two eyes (one of which had had silicone oil injected), rebleeding in one eye and severe post-vitrectomy scleritis in one eye, ${ }^{18}$ three eyes required enucleation. Of the 49 eyes with vitreous haemorrhage but no rubeosis or extramacular traction detachment preoperatively, 46 were immediately stabilised (94\%). No aphakic or pseudo-aphakic eye required a postvitrectomy vitreous washout.

The results of vitrectomy in the macular detachment series are presented in Figures 3 and 4 which indicate that immediate stabilisation was achieved in 35 out of the 81 eyes $(43 \%)$. Ultimate stabilisation was achieved in a further 12 eyes using a variety of post-vit- 


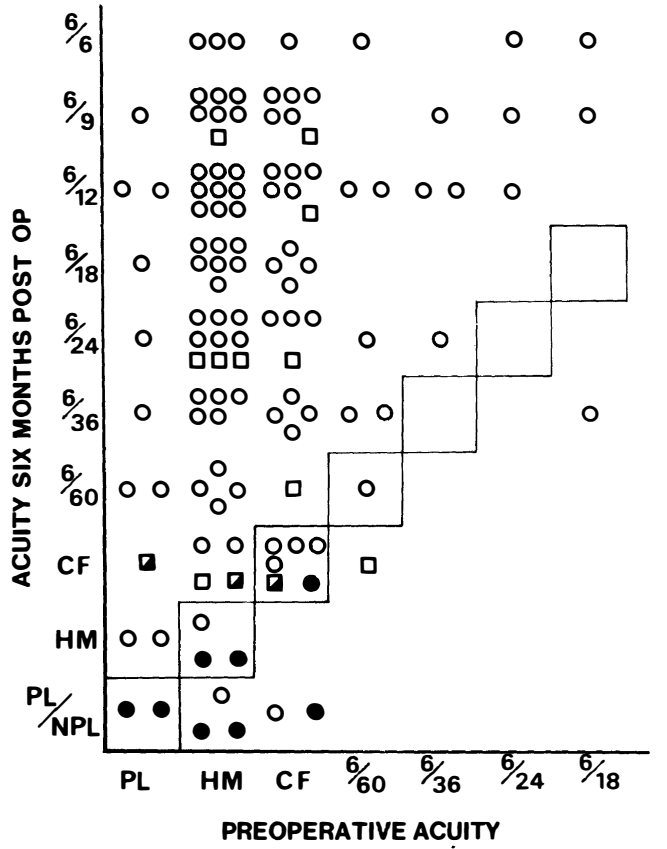

Fig. 1. Scattergram comparing preoperative acuity and final acuity (i.e. six months postvitrectomy or six months after further surgical intervention) in 119 eyes undergoing microsurgery for diabetic vitreous haemorrhage. Each symbol represents one eye; symbols above the oblique line of boxes represent eyes with improved vision. Open circles = eyes immediately stabilised. Open boxes = eyes ultimately stabilised. Half-closed boxes $=$ unstable eyes. Closed circles $=$ eyes with failed surgery.

rectomy procedures including removal of silicone oil in nine eyes (three of which also required cataract extraction), scleral buckling (one eye), by laser photocoagulation (one eye), and laser photocoagulation and vitreous washout (one eye). All 15 'unstable' eyes had been otherwise successfully treated but retained silicone oil in-situ at six months postvitrectomy. The causes of failure in 19 eyes included abandonment of surgery because of untreatable pathology in three eyes (two of which had vitreous haemorrhage and macular detachment by ultrasound and thus no direct visualisation of the surgical configuration), retinal detachment in 13 eyes (six of which had severe reparative epiretinal fibrosis and three of which had severe rubeosis), reparative epiretinal fibrosis and retro-silicone oil neovascularisation in two eyes, and localised macular distortion from reparative fibrosis in one eye.
Overall two-thirds of the eyes (133/200 eyes) operated in this consecutive series underwent 'definitive' surgery, i.e. they required no further procedures in order to achieve stabilisation at six months post-vitrectomy. Added to this, a further 22 eyes became stabilised at six months by virtue of further interventions (155/200 eyes stabilised in all). Twenty-seven eyes were surgical failures.

\section{(ii) Visual Outcome}

The functional outcomes in the haemorrhage and macular detachment groups are represented in Figures 1-4. Summarising the comparison between pre-operative and six-month post-operative vision in the vitreous haemorrhage group (Figure 1), 100 out of 119 eyes $(84 \%)$ had improved vision, 11 eyes $(9 \%)$ had the same vision and eight eyes $(7 \%)$ had worse vision as a result of surgery. In 87 eyes $(73 \%)$, postoperative vision was $6 / 36$ or better. Comparison of vision six months postvitrectomy with that in the fellow eye (Figure 2) shows that the operated eye had better vision than the fellow eye in 67 instances $(56 \%)$, there was equal vision in each eye in 14

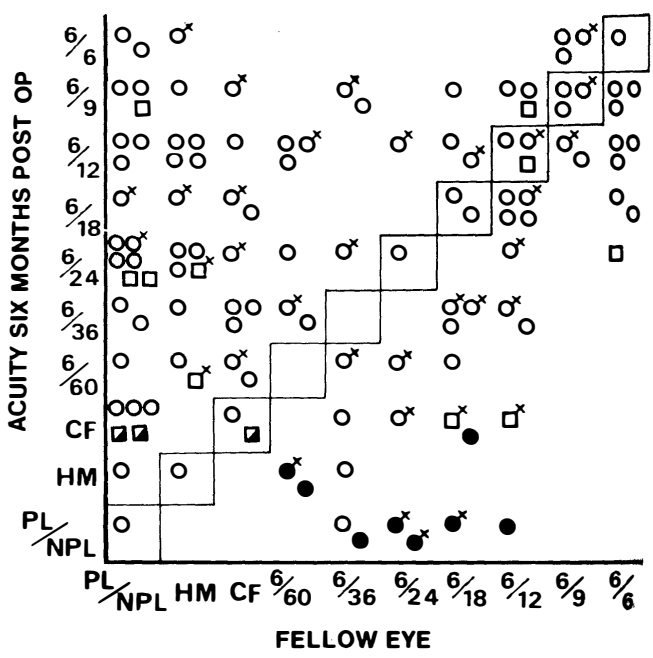

Fig. 2. Scattergram comparing final acuity following surgery and acuity in the fellow eye in 119 eyes undergoing microsurgery for diabetic vitreous haemorrhage. Each symbol represents one eye; symbols above the oblique line of boxes represent eyes with better vision in the operated versus the fellow eye. Key to symbols is as in Figure 1; crosses attached to symbols indicate bilateral surgery in this series. 


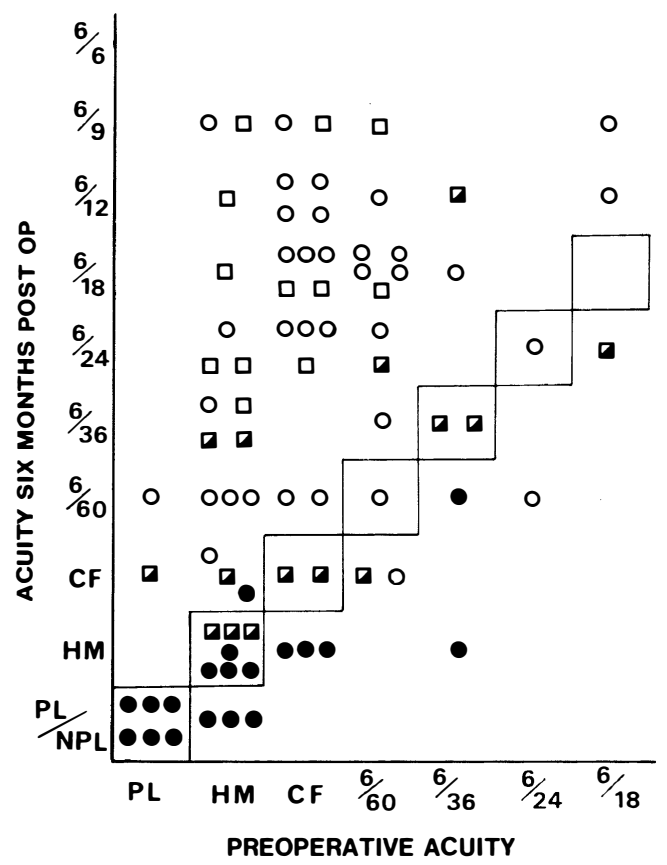

Fig. 3. Scattergram comparing preoperative acuity and final acuity (i.e. six months postvitrectomy or six months after further surgical intervention) in 81 eyes undergoing microsurgery for diabetic macular detachment. Each symbol represents one eye; symbols above the oblique line of boxes represent eyes with improved vision. Key to symbols as in Figure 1.

instances $(12 \%)$ while, in 38 eyes $(32 \%)$, the operated eye had worse vision than that in the fellow eye. However, taking eyes with equal or worse vision than that in the fellow eye, the operated eye made a contribution to binocular function (by virtue of at least $6 / 36$ vision in each eye) in 31 eyes, so vitrectomy for vitreous haemorrhage was considered 'worthwhile' in a total of 97/119 instances $(82 \%)$, i.e. excluding any patient with less than finger counting vision overall.

Summarising the visual results in the macular detachment group (Figure 3), 50 out of 81 eyes $(62 \%)$ were improved, $15(19 \%)$ were unchanged and $16(20 \%)$ were worse following surgery. A vision of $6 / 36$ or better was achieved in 44 eyes $(54 \%)$ with preoperative macular detachment. Of these 81 eyes, 32 eyes $(40 \%)$ had better vision than in the fellow eye at six months following surgery, 11 eyes $(14 \%)$ had equal vision and 38 eyes $(47 \%)$ had worse vision than that in the fellow eye (Figure 4). However, 22 of the eyes with equal or worse vision than the fellow eye nevertheless made a significant contribution to binocular function (i.e. presence of at least $6 / 36$ vision in each eye) so surgery was considered appropriate by this criterion in 50/81 eyes overall $(62 \%)$, i.e. excluding any patient with less than finger counting vision overall.

\section{Discussion}

From the standpoint of the technical outcome, this series of diabetic vitrectomies was 'successful' to a degree determined by the criteria employed. Thus, surgery was definitive (immediate stabilisation achieved) in $82 \%$ of the haemorrhage group and $43 \%$ of the macular detachment group, and some degree of technical success (i.e. absence of failure) was enjoyed in $93 \%$ of the haemorrhage group and $77 \%$ of the macular detachment group. Furthermore, achievement of at least $6 / 36$ vision at six months post-operatively was recorded in $73 \%$ of the haemorrhage series and $54 \%$ of the macular detachment group. The technical outcomes might have been improved by more experienced or technically competent surgeons (either within the surgical firm or otherwise), by use of more

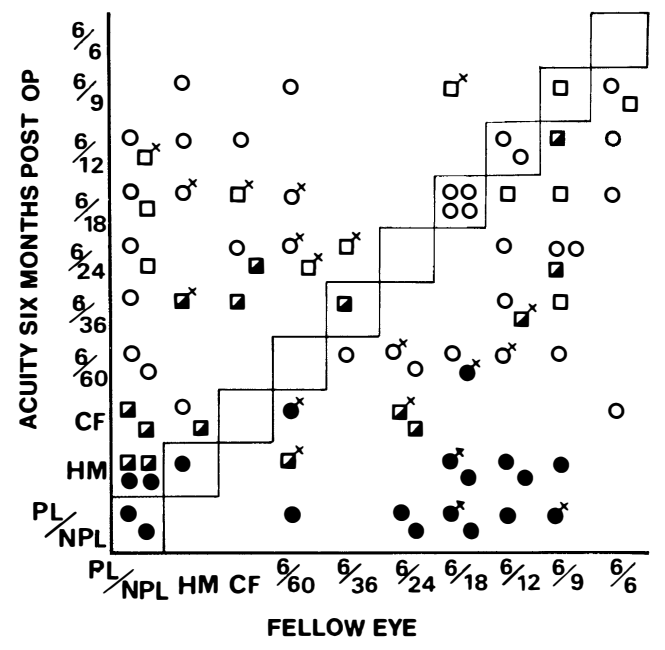

Fig. 4. Scattergram comparing final acuity following surgery and acuity in the fellow eye in 81 eyes undergoing microsurgery for diabetic macular detachment. Each symbol represents one eye; symbols above the oblique line of boxes represent eyes with better vision in the operated versus the fellow eye. Key to symbols as in Figure 1; crosses attached to symbols indicate bilateral surgery in this series. 
sophisticated surgical technology (e.g. longer wavelength endolaser) or by use of more advanced pharmacological aids to vitrectomy (such as thrombin to limit intra-operative bleeding or tissue plasminogen activator for post-operative fibrin preceding fibrosis). The results were, however, consistent with a sustainable surgical rationale. The overall functional results might have been less satisfactory had not patients with a putative poor visual prognosis (whatever the technical outcome of surgery) been excluded during case selection.

Determination of the appropriateness of case selection is a difficult and potentially invidious exercise in any form of surgery. Yet cost:benefit as well as risk:benefit analysis is essential in any cash-limited health provision, the only question being 'where does one draw the line?' Notwithstanding the tremendous emotional pressure to try to restore some vision in premature babies with retinopathy of prematurity, many would consider that the appropriateness of such surgery has not yet been established, bearing in mind the modest techrical outcomes and poor visual outcomes. Furthermore, excepting 'only eyes', much of the vitreous surgery performed for 'proliferative vitreoretinopathy' would not withstand critical appraisal bearing in mind the poor visual outcomes following, albeit, ultimately technically successful surgery. Patients with severe diabetic eye disease warrant the most careful case selection in view of their multiple other deficits and the likelihood of similar (if not simultaneous) damage to both eyes. On the one hand, it has been suggested that surgery for unilateral vitreous haemorrhage is inappropriate $^{12}$ in view of the risk of serious sequelae such as retrolental neovascularisation and loss of the eye (though this presupposes that vitreous haemorrhages can be safely left indefinitely and also denies patients binocular function). On the other hand, denying a patient surgery for a severe combined rhegmatogenous and table-top traction retinal detachment when the other eye is fully stabilised and functional following scatter photocoagulation needs no justification.

The 'appropriateness' of surgery in this series has been quantified using arbitrary and retrospective criteria albeit ones which might have some merit in respect of comparative audit exercises. Case selection was considered appropriate in $82 \%$ of the vitreous haemorrhage group and $62 \%$ of the macular detachment group based on a comparison with the fellow eye and achievement of at least finger counting vision plus either better vision in the operated compared with the fellow eye or, if not, then a significant contribution to binocular vision (i.e. at least $6 / 36$ vision in each eye). The major factors confounding accurate prognostication before embarking on surgery were the degree of macular ischaemia, the potential recovery of macular function after reattachment and the technical facility of membrano-retinal separation.

In conclusion, two comments about laser therapy. Firstly access to endolaser photocoagulation resulted in $82 \%$ immediate stabilisation in the vitreous haemorrhage series here reported, compared with $74 \%$ in eyes with vitreous haemorrhage undergoing vitrectomy and Xenon arc endophotocoagulation between 1981 and 1984 , and $46 \%$ of eyes in $1980 / 81$ prior to the availability of Xenon endophotocoagulation. ${ }^{17}$ There was no change, however, in the rate of ultimate stabilisation $(90 \%, 86 \%$ and $89 \%$ respectively). Nevertheless there is little doubt that intra-operative attention to the underlying cell-biological consequences of retinal ischaemia by using endolaser is not only convenient but is also cost-effective by seeking to ensure 'definitive' surgery. Secondly, much of the surgical activity reported here could have been prevented by adequate and timely biomicroscopic scatter photocoagulation for proliferative diabetic retinopathy. From patients' pre-vitrectomy laser history and the findings at surgery, $37 \%$ of eyes were found to have had minimal laser therapy or none, $61 \%$ of eyes had had 'moderate' photocoagulation and only five eyes had had a 'full' photocoagulation. It is debatable whether all diabetic vitrectomies can truly be prevented by photocoagulation and there may be a place for vitrectomy (as opposed to massive over-photocoagulation) in some eyes with progressive fibrovascular proliferation. It is similarly difficult to judge what increase in screening provision and photocoagulation services would be sufficient to prevent the 
worst excesses of the diabetic fibrovascular process. It must also be acknowledged that many eyes will eventually be complicated by vitreous haemorrhages during progressive posterior vitreous detachment however successfully regression of neovascularisation is judged to have occurred following scatter laser treatment.

Key words: diabetic vitrectomy; medical audit.

\section{References}

${ }^{1}$ Carter JB, Michels RG, Glaser BM, de Bustros S: Iatrogenic retinal breaks complicating pars plana vitrectomy. Ophthalmol 1990; 97: 848-54.

${ }^{2}$ Stefansson E, Novack RL, Hatchell DL: Vitrectomy prevents retinal hypoxia in branch vein occlusion. Invest Ophthalmol Vis Sci 1990; 31: 284-9.

${ }^{3}$ Rosen PJ, Boulton ME, McLeod D: Effect of different oxygen concentrations on the proliferation of retinal microvascular endothelial cells and pericytes in vitro. Invest Ophthalmol Vis Sci 1989; 30 (Supplement): 316.

${ }^{4}$ Baird A, Esch F, Gospodarowitz D, Guillemin R: Retina and eye-derived endothelial cell growth factors: partial molecular characterisation and identity with acidic and basic fibroblast growth factors. Biochem 1985; 24: 7855-60.

${ }^{5}$ Elstow SF, Schor AM, Weiss JB: Bovine retinal angiogenesis factor is a small molecule. Invest Ophthalmol Vis Sci 1985; 26: 74-9.

${ }^{6}$ Wong HC, Sehmi KS, McLeod D: Abortive neovascular outgrowths discovered during vitrectomy for diabetic vitreous haemorrhage. Von Graefe's Arch Clin Exp Ophthalmol 1989; 227: $237-40$.

${ }^{7}$ Akiba J, Arzabe CW, Trempe CL: Posterior vitreous detachment and neovascularisation in diabetic retinopathy. Ophthalmol 1990; 97: 889-91.

${ }^{8}$ Boulton M, McLeod D, Garner A: Vasoproliferative retinopathies: clinical, morphogenetic and modulatory aspects. Eye 1988; 2: S124-39.

${ }^{9}$ Lewis H, Abrams GW, Williams GA: Anterior hyaloidal fibrovascular proliferation after diabetic vitrectomy. Am J Ophthalmol 1987; 104: 607-13.

${ }^{10}$ Molnar I, Poitry S, Tsacopoulos M, Gilodi N, Leuenberger PM: Effect of laser photocoagulation on oxygenation of the retina in miniature pigs. Invest Ophthalmol Vis Sci 1985; 26: 1410-4.

${ }^{11}$ Barry PJ, Hiscott PS, Grierson I, Marshall J, McLeod D: Reparative epiretinal fibrosis after diabetic vitrectomy. Trans Ophthalmol Soc UK 1985; 104: 285-96.

${ }^{12}$ Charles S: Vitreous microsurgery. Baltimore: Williams \& Wilkins, 1981

${ }^{13}$ McLeod D and James CR: Viscodelamination at the vitreoretinal juncture in severe diabetic eye disease. Br J Ophthalmol 1988; 72: 413-9.

${ }^{14}$ Williams DF, Williams GA, Hartz A, Meiler WF, Abrams GA, Aaberg TM: Results of vitrectomy for diabetic traction retinal detachment using the en bloc excision technique. Ophthalmol 1989; 96: 752-8.

${ }^{15}$ McLeod D: Use of silicone oil in diabetic vitrectomy. Internat Ophthalmol 1985; 8: 104-5.

${ }^{16}$ McLeod D: Silicone-oil injection during closed microsurgery for diabetic retinal detachment. Von Graefe's Arch Clin Exp Ophthalmol 1986; 224: 55-9.

${ }^{17}$ Ficker L, Passani F, Leaver PK, McLeod D: Xenonarc endophotocoagulation during vitrectomy for diabetic vitreous haemorrhage. Von Graefe's Arch Clin Exp Ophthalmol 1986; 224: 423-7.

${ }^{18}$ Young RD, Powell J, Watson PG: Ultrastructural changes in scleral proteoglycans precede destruction of the collagen fibril matrix in necrotising scleritis. Histopathol 1988; 12: 75-84. 